\title{
A novel measurement of marginal Alfvén Eigenmode stability during high power auxiliary heating in JET
}

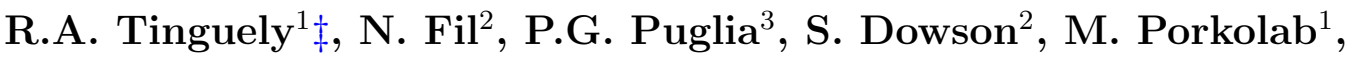 \\ V. Guillemot ${ }^{4}$, M. Podestà ${ }^{5}$, M. Baruzzo ${ }^{2}$, R. Dumont ${ }^{6}$, A. Fasoli ${ }^{3}$, \\ M. Fitzgerald ${ }^{2}$, Ye.O. Kazakov ${ }^{7}$, M.F.F. Nave ${ }^{8}$, M. Nocente ${ }^{9}{ }^{10}$, \\ J. Ongena ${ }^{7}$, S.E. Sharapov ${ }^{2}$, Ž. Štancar ${ }^{11}$, and JET Contributors* \\ ${ }^{1}$ Plasma Science and Fusion Center, Massachusetts Institute of Technology, Cambridge, \\ MA, USA \\ ${ }^{2}$ Culham Centre for Fusion Energy, Culham Science Centre, Abingdon, UK \\ ${ }^{3}$ Ecole Polytechnique Fédérale de Lausanne (EPFL), Swiss Plasma Center (SPC), \\ CH-1015 Lausanne, Switzerland \\ ${ }^{4}$ Ecole Supérieure de Physique et de Chimie Industrielles de la Ville de Paris, 75231 Paris \\ Cedex 05, France \\ ${ }^{5}$ Princeton Plasma Physics Laboratory, Princeton, NJ, USA \\ ${ }^{6}$ CEA, IRFM, F-13108 Saint-Paul-lez-Durance, France \\ ${ }^{7}$ Laboratory for Plasma Physics, LPP-ERM/KMS, TEC Partner, 1000 Brussels, Belgium \\ ${ }^{8}$ Instituto de Plasmas e Fusão Nuclear, Instituto Superior Técnico, Univ. de Lisboa, \\ Lisbon, Portugal \\ ${ }^{9}$ Dipartimento di Fisica, Universitá di Milano-Bicocca, 20126 Milan, Italy \\ ${ }^{10}$ Institute for Plasma Science and Technology, National Research Council, 20125, Milan, \\ Italy \\ ${ }^{11}$ Jožef Stefan Institute, Ljubljana, Slovenia \\ * See the author list of "Overview of JET results for optimising ITER operation" by \\ J. Mailloux et al to be published in Nuclear Fusion special issue: Overview and Summary \\ Papers from the 28th Fusion Energy Conference (Nice, France, 10-15 May 2021)
}

\begin{abstract}
.
The interaction of Alfvén Eigenmodes (AEs) and energetic particles is one of many important factors determining the success of future tokamaks. In JET, eight in-vessel antennas were installed to actively probe stable AEs with frequencies ranging $25-250 \mathrm{kHz}$ and toroidal mode numbers $|n|<20$. During the 2019-2020 deuterium campaign, almost 7500 resonances and their frequencies $f_{0}$, net damping rates $\gamma<0$, and toroidal mode numbers were measured in almost 800 plasma discharges. From a statistical analysis of this database, continuum and radiative damping are inferred to increase with edge safety factor, edge magnetic shear, and when including non-ideal effects. Both stable AE observations and their associated damping rates are found to decrease with $|n|$. Active antenna excitation is also found to be ineffective in H-mode as opposed to L-mode; this is likely due to the increased edge density gradient's effect on accessibility and ELM-related noise's impact on mode identification. A novel measurement is reported of a marginally stable, edge-localized Ellipticity-induced AE probed by the antennas during high-power auxiliary heating (ICRH
\end{abstract}

$\ddagger$ Author to whom correspondence should be addressed: rating@mit.edu 
and NBI) up to $25 \mathrm{MW}$. NOVA-K kinetic-MHD simulations show good agreement with experimental measurements of $f_{0}, \gamma$, and $n$, indicating the dominance of continuum and electron Landau damping in this case. Similar experimental and computational studies are planned for the recent hydrogen and ongoing tritium campaigns, in preparation for the upcoming DT campaign.

Keywords: Alfvén Eigenmode, stability, Neutral Beam Injection, Ion Cyclotron Resonance Heating

\section{Introduction}

The Alfvén Eigenmode Active Diagnostic (AEAD) actively probes, or excites, stable Alfvén Eigenmodes (AEs) in JET tokamak plasmas [1-3]. The importance of these AE stability measurements - i.e. frequencies $\omega_{0}=2 \pi f_{0}$, net damping rates $\gamma<0$, and toroidal mode numbers $n$ - cannot be overstated. First, they provide a direct experimental comparison with net growth rates calculated from theory and simulation, from which the contributions of different driving and damping mechanisms can be assessed. Of particular interest is the measurement of alpha particle drive, which is a primary goal of energetic particle (EP) experiments [4] and AEAD operation in the upcoming JET DT campaign [5]. Importantly, the AEAD may be the only diagnostic capable of assessing this drive if the alpha population is insufficient to destabilize AEs. Finally, a better understanding of AE stability will improve projections of EP-driven AEs and the resulting AE-induced EP transport in next-step tokamaks, such as ITER and SPARC, and in future fusion pilot plants.

The JET AEAD comprises two in-vessel sets of four toroidally spaced antennas positioned below the outboard midplane and on opposite sides of the torus [2]. Six amplifiers power six (of eight) antennas with currents $\sim 6 \mathrm{~A}$ each; the resulting magnetic perturbation has magnitude $|\delta B / B|<10^{-3}$ at the plasma edge. Independent phasing of the antennas allows power to be injected into a spectrum of toroidal mode numbers, $|n|<20$ [3]. As the scanning antenna frequency passes through that of a stable AE, the plasma resonates like a driven, weakly damped harmonic oscillator [1], and the frequency-filtered magnetic response

- obtained from a toroidal array of fast magnetic probes - determines $f_{0}, \gamma$, and $n$ [6]. The external antennas are more likely to excite AEs near the plasma edge than in the core; these often include Global, Toroidicity-induced, and Ellipticity-induced AEs (GAEs, TAEs, and EAEs, respectively) [7].

This paper reports on recent progress in experimental and computational studies of $\mathrm{AE}$ stability with the AEAD. The organization is as follows: Section 2 gives a brief review of past results and motivates this study. Then, in Section 3, an expanded database of AE and plasma parameters is presented, along with statistically significant trends related to AE physics. Section 4 focuses on the novel measurement of a stable AE probed by the AEAD during high-power external heating of a D- ${ }^{3} \mathrm{He}$ plasma, and experimental results are compared with kinetic-MHD simulations in Section 5. Finally, a summary is given in Section 6. 


\section{Motivation}

Many past studies have analyzed stable AEs in JET with the AEAD [1-3,6-37], including measurements made during the 1997 JET DT campaign [14]. However, at the time, the saddle coil system was ineffective in probing stable AEs during high performance phases in $\mathrm{X}$-point magnetic configuration $[10,14,17,18]$. Since then, there have been upgrades to the system [2], including the independent powering and phasing of the antennas [3], with the main aim of measuring AE stability in the upcoming JET DT campaign [5]. Thus, it is of interest to map the operational space of the current system, compare with past experimental results, and optimize for near-future studies.

The focus of this paper is the demonstration of the AEAD's ability to excite stable AEs in high-power, high-performance plasmas. First, trends in the net damping rate with auxiliary heating and confinement regime are presented in the next section. Then, in Sections 4 and 5, a novel AE stability measurement is investigated in depth for one high-power D- ${ }^{3} \mathrm{He}$ plasma discharge. The observation of this stable AE was actually surprising in several ways: First, significant ion Landau damping was expected from the Neutral Beam Injection (NBI) heating power $P_{\mathrm{NBI}} \approx 20 \mathrm{MW}$ during the discharge since significant NBI damping of AEs has been observed before in many devices, including JT-60U [38], TFTR [13], and JET [4, 10, 20]. The plasma was also in X-point configuration, which makes stable AE detection even less likely $[7,39]$. Yet we were able to track a stable AE in real time, during X-point configuration, and at the highest external heating power to-date $(\sim 25 \mathrm{MW})$. Such a measurement gives the authors confidence in overcoming evidence of limited diagnostic efficiency for successful operation in DT.

\section{Database studies of stable Alfvén Eigenmodes}

This section presents a statistical analysis of thousands of stable AEs collected in the recent 2019-2020 JET deuterium campaign. The AEAD was operated on almost 800 plasma discharges, from which a database of almost 7500 stable AE measurements was assembled. Note that this database is actually an expansion of that reported earlier in [6] and [7] because new data were acquired after their publications.

In the following analyses and unless otherwise stated, data are restricted to normalized damping rates $-\gamma / \omega_{0} \leq 6 \%$, uncertainties $\Delta f_{0}<1 \mathrm{kHz}$ and $\Delta\left(\gamma / \omega_{0}\right)<1 \%$, X-point magnetic configuration, and heating powers $P_{\mathrm{NBI}}<7 \mathrm{MW}$ and $P_{\mathrm{ICRH}}<7 \mathrm{MW}$ from Ion Cyclotron Resonance Heating (ICRH). The last two bounds are motivated by the sometimesoverwhelming pick-up in magnetics data which can lead to misidentification of AEs [27]. Thus, resonances observed during $P_{\mathrm{NBI}}, P_{\mathrm{ICRH}}>7 \mathrm{MW}$ require closer inspection, as done in Sections 4 and 5 . 


\subsection{Statistical analyses of damping rates}

Two recent works have analyzed stable AEs from this database: The damping rate was observed to increase linearly with the edge safety factor $q_{95}$ (see Fig. 5a in [6]). This was attributed to an increase in continuum damping as the AE continuum gap closes at the plasma edge, which was also seen before in JET [33]. The damping rate was also noted to increase rapidly and nonlinearly with the edge magnetic shear $s_{95}$ (see Fig. 6a in [7]), likely due to both continuum and radiative damping. This result was in agreement with past works $[10,14,17,18,23,33]$. These trends are confirmed to have statistical significance in Table 1, which reports the linear correlation $r_{\mathrm{w}}\left(-\gamma / \omega_{0}, x\right)$ of the normalized damping rate, weighted by its inverse variance $\Delta\left(\gamma / \omega_{0}\right)^{-2}$, with various parameters $x$. Here, magnitudes $\left|r_{\mathrm{w}}\right| \geq 0.5$ are considered significant.

Table 1: Weighted linear correlations of normalized damping rate. Subscripts 0 and 95 refer to parameters evaluated at normalized poloidal flux values $\psi_{\mathrm{N}}=0$ and 0.95 , respectively. Note that the correlation with NBI heating power is restricted to values $P_{\mathrm{NBI}}>0$ during no $\mathrm{ICRH}\left(P_{\mathrm{ICRH}}=0\right)$, and vice versa. Bold values, $\left|r_{\mathrm{w}}\right| \geq 0.5$, are considered significant.

\begin{tabular}{cc}
\hline Parameter $x$ & $r_{\mathrm{w}}\left(-\gamma / \omega_{0}, x\right)$ \\
\hline$q_{0}$ & -0.11 \\
$q_{95}$ & $\mathbf{0 . 5 4}$ \\
$s_{95}$ & $\mathbf{0 . 5 7}$ \\
$\lambda$ & $\mathbf{0 . 6 9}$ \\
$B_{0}$ & -0.12 \\
$T_{\mathrm{e} 0}$ & -0.01 \\
$n_{\mathrm{e} 0}$ & -0.20 \\
$n_{\mathrm{e} 95}$ & -0.34 \\
$\nabla n_{\mathrm{e} 95}$ & 0.29 \\
$P_{\mathrm{NBI}}$ & 0.28 \\
$P_{\mathrm{ICRH}}$ & -0.09 \\
\hline
\end{tabular}

The highest correlation is with the so-called non-ideal parameter $\lambda=q_{95} s_{95} \sqrt{T_{\mathrm{e} 0}} / B_{0}$ $[32,40]$, where $T_{\mathrm{e} 0}$ and $B_{0}$ are the on-axis values of the electron temperature and toroidal magnetic field, respectively. Note that no correlation is observed with $T_{\mathrm{e} 0}$ and $B_{0}$ individually. The non-ideal parameter is key in the theory of radiative damping [41,42], and therefore $\lambda$ is a better indicator of its impact than $q_{95}$ or $s_{95}$ alone. Previous computational efforts with a variety of MHD, kinetic, and gyrokinetic codes [43] had also identified enhanced radiative damping with increasing temperature and hence larger gyro-radius effects. All data points are shown in Fig. 1a with partial transparency, and a clear linear trend is observed in the highest-density (i.e. the darkest) region. At present, the divergence from this trend at low $\lambda$ $\left(<600 \mathrm{eV}^{1 / 2} / \mathrm{T}\right)$ is not well understood.

Correlations with NBI and ICRH powers are also given in Table 1. Here, data are 


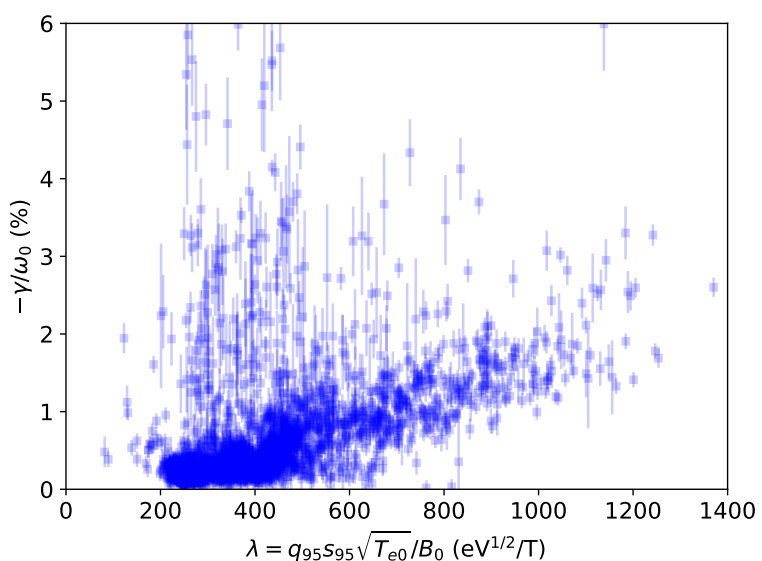

(a)



(b)

Figure 1: Normalized damping rate vs (a) non-ideal parameter and (b) NBI power, with uncertainties as error bars. A linear fit is overlaid in (b).

restricted to non-zero values of heating power since wide variation in the damping rate is observed with no external heating. Additionally, only one external heating source is considered at a time, i.e. $P_{\mathrm{NBI}}>0$ when $P_{\mathrm{ICRH}}=0$, and vice versa. Even with these restrictions, there are still hundreds of data points to analyze. While the reported correlation with $P_{\text {ICRH }}$ is poor, that with $P_{\mathrm{NBI}}$ is moderate, and a linear fit to the damping rate indicates increased damping with NBI (also observed in [20]). This is expected on JET because NBI fast ion energies are $<100 \mathrm{keV}$, which is typically lower than the Alfvén speed $v_{\mathrm{A}}$.

Damping rate data vs NBI power are shown in Fig. $1 \mathrm{~b}$, with clusters around $P_{\mathrm{NBI}} \approx$ $1,2,3,4 \mathrm{MW}$ resulting from the discrete steps in power delivered by individual injectors. The calculated slope is low, only $0.042 \% / \mathrm{MW}$, extrapolating to $-\gamma / \omega_{0} \approx 1.5 \%$ at $P_{\mathrm{NBI}} \approx 30 \mathrm{MW}$. Because the AEAD primarily probes stable AEs localized near the plasma edge while NBI power is mostly deposited in the core, this extrapolation is realistically a lower bound on NBI ion Landau damping. Yet, it is important to note that there is sufficient scatter in the data, and the trend could be caused by conflating factors, though not $P_{\mathrm{ICRH}}$.

\subsection{Trends related to operational scenarios}

Investigations of the AEAD operational space are also important, especially as they relate to high-power, high-performance scenarios and the upcoming JET DT campaign. In [6], it was reported that the AEAD's efficiency of resonantly exciting stable AEs decreased with increasing plasma current and external heating power. A thorough study of AEAD-plasma coupling [7] also showed reduced efficiency in X-point (i.e. diverted) magnetic configuration compared to limiter configuration - consistent with the $q_{95}$ and $s_{95}$ trends above - as well as with increased plasma-antenna separation.

Recently, the EUROfusion JET-ILW pedestal database [44] was compared with the 
stable AE database: Interestingly, of the approximately 500 stable AEs measured in over 80 plasma discharges common to both databases, none were observed during H-mode periods. This confirms, with relatively high confidence $(\mathrm{p}$-value $=0.076)$, that the AEAD is inefficient in probing stable AEs during $\mathrm{H}$-mode. One probable cause is the effect of the density pedestal on the AE continuum. In fact, recent modeling work [39] highlighted the impact of the edge density profile on AE continua as well as AEAD accessibility. Yet, there still exist some stable AE measurements during high performance operation, as explored in Section 4.

To investigate further the effect of edge density conditions - and complement that of edge magnetic conditions $q_{95}$ and $s_{95}$ above - the edge electron density $n_{\mathrm{e} 95}=n_{\mathrm{e}}\left(\psi_{\mathrm{N}}=0.95\right)$, obtained from Thomson Scattering, and its gradient $\nabla n_{\mathrm{e} 95}=\mathrm{d} n_{\mathrm{e}} /\left.\mathrm{d} \psi_{\mathrm{N}}\right|_{\psi_{\mathrm{N}}=0.95}$ were also analyzed, with $\psi_{\mathrm{N}}$ the normalized poloidal flux. While no "good" correlations, i.e. $\left|r_{\mathrm{w}}\right| \geq 0.5$, are identified (see Table 1), the strongest is found with $n_{\mathrm{e} 95}$.

All data points are shown versus $n_{\mathrm{e} 95}$ in Fig. 2a. Interestingly, there is a clear upper bound on the data, $-\gamma / \omega_{0} \leq\left(n_{\mathrm{e} 95}\right)^{-3 / 2}$, with $\sim 84 \%$ of data falling below this curve. $\oint$ This trend is counterintuitive as the AE gap is expected to close with increasing $n_{\mathrm{e} 95}$ via $v_{\mathrm{A}} \propto n_{\mathrm{e}}^{-1 / 2}$, here assuming $n_{\mathrm{e}} \approx n_{\mathrm{i}}$, so that enhanced continuum damping might be observed. $\|$ However, note that gradient of the continuum contains the term $\mathrm{d} v_{\mathrm{A}} / \mathrm{d} n_{\mathrm{e}} \propto n_{\mathrm{e}}^{-3 / 2}$, matching the bounding curve. This suggests that as $n_{\mathrm{e} 95}$ increases (and $\nabla n_{\mathrm{e} 95}$ becomes steeper), AEAD accessibility is reduced and stable AEs become more difficult to excite, consistent with no observations in H-mode as discussed above.



(a)



(b)

Figure 2: (a) Normalized damping rate vs edge electron density $n_{\mathrm{e} 95}$ with an approximate bounding curve overlaid. (b) Number of stable AE observations (logarithmic) vs toroidal mode number $(|n| \leq 7)$ and normalized damping rate, with uncertainties restricted to $\Delta\left(\gamma / \omega_{0}\right) \leq 0.5 \%$.

It is important to note that reduced AEAD accessibility is not the only issue here; the detection of stable AEs by the fast magnetic probes is also reduced in H-mode. During high-

$\S$ Note that the scaling of this curve is arbitrary.

\| This also depends on the safety factor profile. 
power external heating, significant noise is seen in the magnetics signals (e.g. from ELMs), making stable AEs difficult to identify, even if the AEAD is able to resonate with them. Thus, there are several conflating factors conspiring against successful AEAD operation during Hmode periods.

Finally, the dependencies of the damping rate on toroidal mode number are explored. Figure $2 \mathrm{~b}$ shows the number of stable AE measurements for each estimated $n$ and bin of measured $\gamma / \omega_{0}$. Here, data are restricted to $|n| \leq 7$, consistent with typical unstable AEs observed in JET, and $\left|\Delta\left(\gamma / \omega_{0}\right)\right|<0.5 \%$, allowing a finer grid discretization. As discussed in [6], the high density of $n=0$ values could indicate that these are true Global AEs (GAEs) or that too few magnetic probes were available for a good $n$ estimation.

A general trend of decreasing $\left|\gamma / \omega_{0}\right|$ with $|n|$, also noted in [6], is observed in Fig. $2 \mathrm{~b}$. There are several possible explanations: For a given AE radial location, the mode width decreases as $1 /|n|$ leading to more localized damping, as opposed to more global modes interacting with, say, the continuum. Also, in the presence of fast ions (FI), AE drive increases with $n$ via $n \omega_{* \mathrm{FI}} \propto(n / r) \mathrm{d} p_{\mathrm{FI}} / \mathrm{d} r$, the FI radial pressure gradient. However, an asymmetry for positive and negative $n$ would be expected, which is not easily observed in Fig. 2b. Unfortunately, even within a database of almost 7500 stable AEs, no $\pm n$ pair exists with sufficiently similar plasma or FI conditions to estimate $\mathrm{d} p_{\mathrm{FI}} / \mathrm{d} r$, as suggested in [18].

\section{Experimental measurements of marginal AE stability during high-power heating}

This section reports on a novel measurement of AE stability during JPN 94703 with high auxiliary heating power, $P_{\mathrm{NBI}}+P_{\mathrm{ICRH}} \approx 25 \mathrm{MW}$. This pulse was part of the three-ion-heating scenario development experiments at JET relevant to the upcoming JET DT campaign as well as ICRH in ITER $[5,45,46]$. Experimental results are presented here, and comparisons with kinetic-MHD simulations are given in Section 5.

Time traces of plasma parameters for JPN 94703 are shown in Fig. 3a, with the time range of interest, $t=8-12 \mathrm{~s}$, shaded. Flattop parameters are $B_{0}=3.7 \mathrm{~T}, I_{\mathrm{p}}=2.5 \mathrm{MA}$, $n_{\mathrm{e} 0} \approx 8 \times 10^{19} \mathrm{~m}^{-3}$, and $T_{\mathrm{e} 0} \approx 5.3 \mathrm{keV}$. Auxiliary heating are $P_{\mathrm{NBI}} \approx 19-21 \mathrm{MW}$

and $P_{\mathrm{ICRH}} \approx 4.4 \mathrm{MW}$ from $t=8-11 \mathrm{~s}$. The concentration of ${ }^{3} \mathrm{He}$ is relatively high, $n_{\mathrm{He} 3} / n_{\mathrm{e}} \approx 23 \%$, as part of the D-(D $\left.\mathrm{DBI}\right)-{ }^{3} \mathrm{He}$ heating scheme [47].

Plasma profiles are shown for one time, $t=8.5 \mathrm{~s}$, in Fig. $3 \mathrm{~b}$ as a function of the normalized poloidal flux $\psi_{\mathrm{N}}$. Two $q$-profiles from EFIT [48] are shown: One is constrained by the fitted kinetic profiles from TRANSP [49-51], which match Thomson Scattering $n_{\mathrm{e}}$ and $T_{\mathrm{e}}$ data well. (Here, equal electron and ion temperatures, $T_{\mathrm{e}}=T_{\mathrm{i}}$, are assumed.) The other $q$-profile is additionally constrained by polarimetry measurements and agrees within $\sim 10 \%$. Note that this time-slice is between two $T_{\mathrm{e}}$ sawtooth crashes at $t \approx 8.33 \mathrm{~s}$ and $8.55 \mathrm{~s}$, and electron cyclotron emission data indicate that the inversion radius is at $R \approx 3.13 \mathrm{~m}$ $\left(\psi_{\mathrm{N}} \approx 0.03\right)$. Thus, the $q$-profile fit with polarimetry is likely more accurate; however, as will be seen in the next section, the AE analysis is fairly robust to these uncertainties in $q$. 
Finally, rotation data is obtained from ${ }^{3} \mathrm{He}$ charge exchange spectroscopy.
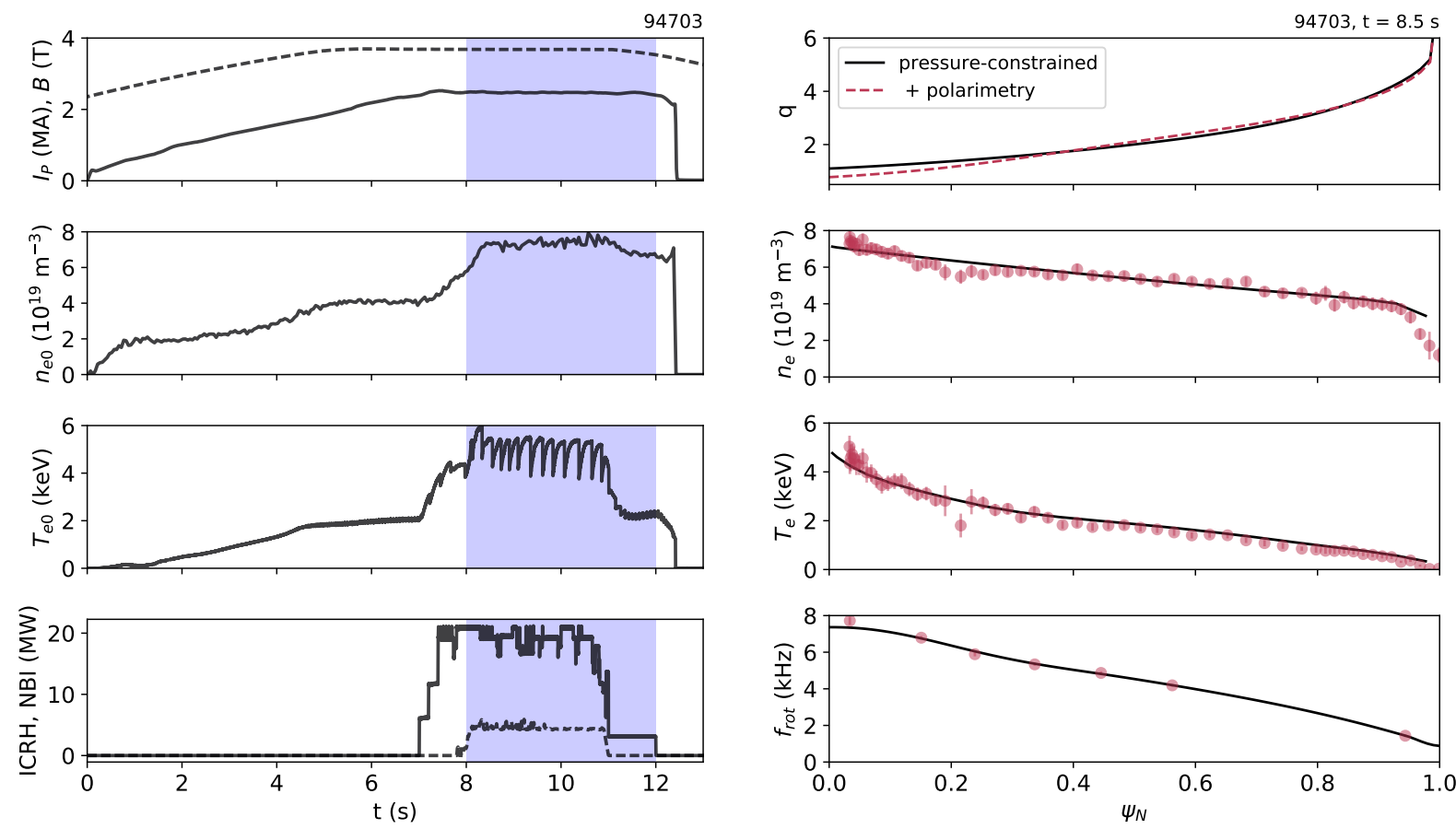

(a)

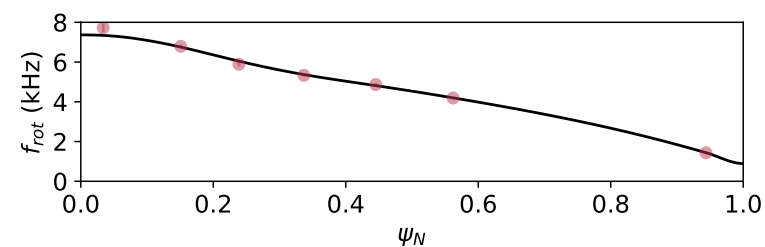

(b)

Figure 3: (a) Plasma parameters for JPN 94703: toroidal magnetic field (dashed), plasma current (solid), central electron density from Thomson Scattering (TS) and temperature from Electron Cyclotron Emission, and heating powers from NBI (solid) and ICRH (dashed). A stable AE was tracked during the shaded time interval. (b) Profiles at $t=8.5 \mathrm{~s}$ : safety factor from EFIT constrained by pressure only (solid) and additionally polarimetry (dashed), electron density and temperature from TS, and rotation frequency from ${ }^{3} \mathrm{He}$ charge exchange. Experimental data are shown as circles with uncertainties as error bars, while solid lines are fitted data.

A stable AE was tracked in real time during the high heating power phase. This can be seen in the Fourier decomposition of magnetic signals in Fig. 4a. The triangular waveform is the scanning AEAD frequency, $f=125-250 \mathrm{kHz}$. The antenna phasing was such that power was injected primarily into odd toroidal mode numbers (i.e. $|n|=1,3,5, \ldots$ ) which is confirmed by the mode number analysis of toroidally distributed magnetic probes' data, showing primarily $n=3$ (magenta). Around $t=8.5 \mathrm{~s}$, a stable AE is detected by the AEAD at $f_{0} \approx 235 \mathrm{kHz}$, and the real-time monitoring system quickly changes the scan direction to track the mode. Unfortunately, tracking is "lost" just before $t \approx 9 \mathrm{~s}$, and the scan continues downward in frequency. A marginally unstable AE is then seen between $t \approx 9-10 \mathrm{~s}$ with $f_{0} \approx 235 \mathrm{kHz}$ and a mix of $n=0$ (grey) and $n=5$ (cyan). The fact that the mode is unstable, even with a very small amplitude, could be the reason why the AEAD did not identify it. The AEAD finds the stable mode again at $t \approx 10.5 \mathrm{~s}$ and then tracks until $t \approx 12 \mathrm{~s}$ when $f_{0} \approx 250 \mathrm{kHz}$. 
The measured stable AE parameters are shown in Fig. 4b. Here, the same AE resonance must be detected by at least three (of fourteen) magnetic probes to be considered "good." Characteristic peaks in the magnetic response (summed from all probe amplitudes) are observed, though they are easier to identify by eye after $t>10 \mathrm{~s}$ as external heating is lowered. The resonant frequency of the $\mathrm{AE}$ is relatively smooth in time, and the gap in measurements in Fig. 4b is easily connected by the marginally stable AE visible in Fig. 4a during that interval. The damping rate is also relatively constant in time, ranging from $-\gamma / \omega_{0} \approx 0.17 \%-0.45 \%$ with mean value $-\gamma / \omega_{0} \approx 0.28 \%(-\gamma \approx 1 \mathrm{kHz})$, indicating marginal stability. Thus, only a small increase in AE drive was needed to cause the transition from stability to instability in the interval $t \approx 9-10 \mathrm{~s}$. Finally, note how $\gamma / \omega_{0}$ does not change much as NBI power is reduced from $t \approx 10.5-11 \mathrm{~s}$.

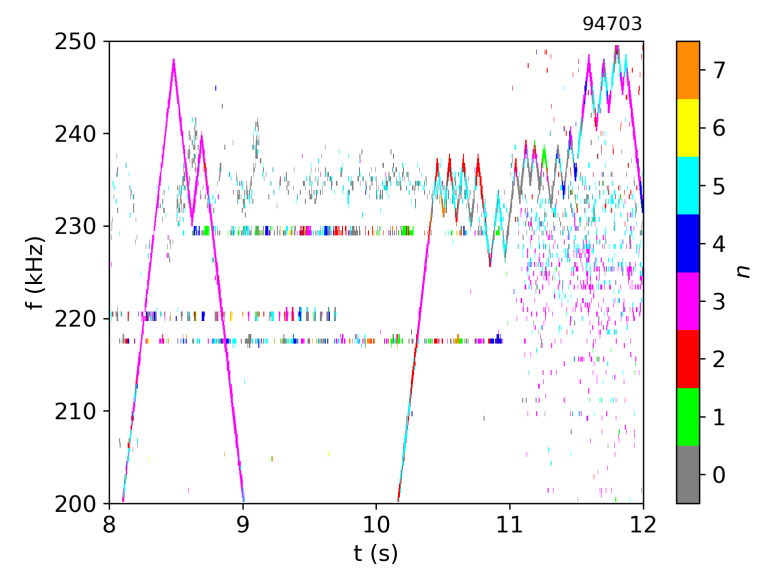

(a)
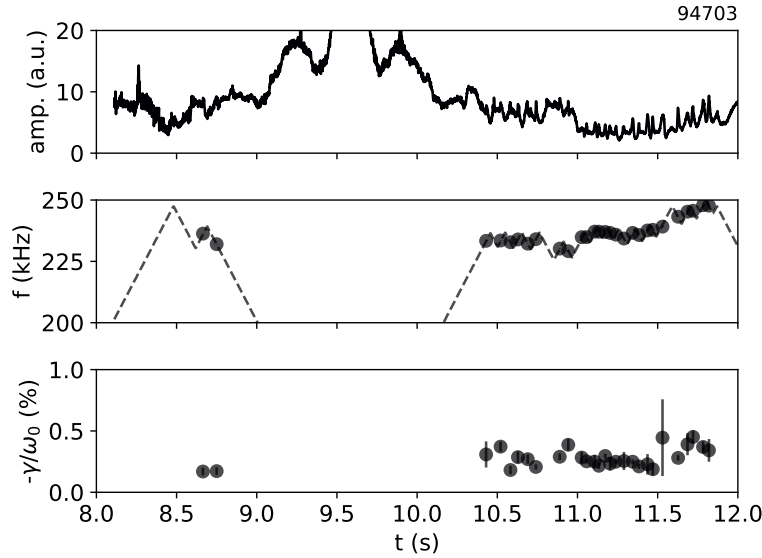

(b)

Figure 4: (a) Fourier decomposition of magnetics data with toroidal mode number analysis. (b) Stable AE resonance measurements: the magnetic response amplitude (summed over all probes), AEAD (dashed) and resonant frequencies (circles), and normalized damping rates with uncertainties as error bars.

Also note in Fig. 3b that the edge density is $n_{\mathrm{e} 95} \approx 4 \times 10^{19} \mathrm{~m}^{-3}$, which is higher than all data in the database of the previous section (see Fig. 2a). 9 Comparing with the database trend, it is consistent - and perhaps fortuitous - that a marginally stable AE is observed in this pulse. A stable AE with higher damping rate may not have been measured at all. In fact, from $t=7.5-8.6 \mathrm{~s}$, the plasma is in L-mode [52], a weak ELM-free H-mode state with no temperature pedestal (see Fig. 3b); the rest of the pulse is confirmed to be in L-mode. Again, this agrees with the conclusion of the previous section that the AEAD has difficulty exciting stable AEs in H-mode.

I The data from JPN 94703 are not actually in the database of Section 3 because of the filter $P_{\mathrm{NBI}}<7$ MW. 


\section{Simulations with the kinetic-MHD code NOVA-K}

While the mode number identification of $n=5$ seems clear in Fig. 4a, this result is unfortunately quite sensitive to the magnetic probes used. In fact, a separate analysis of the AE resonances (not shown) returns a range $|n|=0-5$. Additionally, the mode location is difficult to identify because it is stable and thus not seen in the Fourier analysis of interferometry, reflectometry, or soft X-ray data. That said, the AEAD is known to excite more edge-localized AEs as an external antenna system [7]. Thus, a range of mode numbers, $n=3-6$, is simulated with the NOVA-K kinetic-MHD code [53-55] to assess the existence, mode structure, and stability of AEs. Input profiles are those at $t=8.5 \mathrm{~s}$ (see Fig. $3 \mathrm{~b}$ ), approximately the time of the first stable AE measurement (see Fig. 4b).

In addition to various damping mechanisms, NOVA-K also calculates the contribution to the growth rate from NBI fast ions (FIs), assuming a slowing down distribution. ${ }^{+}$In JPN 94703, deuterium NBI ions, with energies $\sim 100 \mathrm{keV}$, were injected via normal and tangential beams with initial pitches around $v_{\|} / v_{0} \approx 0.44$ and 0.62 , respectively. The deuterium FI distribution function is computed in TRANSP using the NUBEAM and TORIC modules, with NBI and ICRH synergy accounted through the Monte Carlo kick model.

The pitch- and flux-surface-averaged FI distribution is shown in Fig. 5a, while a "slice" at one flux surface $\left(\psi_{\mathrm{N}} \approx 0.5\right)$ is shown in Fig. 5b. The bulk NBI population is clearly seen below $<0.1 \mathrm{MeV}$ with broad extent in radius and pitch. The FI tail, accelerated by ICRH, extends to $\sim 2.5 \mathrm{MeV}$, is primarily core-localized, and exhibits a dominant pitch $v_{\|} / v \approx 0.5$. In contrast to other pulses with the three-ion $\mathrm{D}-\left(\mathrm{D}_{\mathrm{NBI}}\right){ }_{-}{ }^{3} \mathrm{He}$ scheme at lower NBI power [47] with core-localized AEs, the FI population did not destabilize AEs in JPN 94703. As discussed next, the localization of the mode tracked by the AEAD is such that drive from the FI tail would not be expected; therefore, not including its effect on the growth rate is justified in this case. ${ }^{+}$

NOVA-K simulations identify three candidate AEs - i.e. EAEs in the edge gap - with similar frequencies and net damping rates as the experimentally measured mode. Their toroidal mode numbers, resonant frequencies, and breakdown of damping rate contributions are provided in Table 2. Note that the $23 \%{ }^{3} \mathrm{He}$ has not been simulated here, ${ }^{+}$but its expected effect is to increase the Alfvén speed (and hence the plasma-frame AE frequency) by only $\sim 6 \%$, which is approximately the same uncertainty introduced by any fitted profile (see Fig. 3b). In addition, the ion dilution from including multiple thermal ion species would even further reduce the effect of ion Landau damping, discussed in more detail below.

The simulated $n=5$ mode, with resonant frequency $f_{0} \approx 236 \mathrm{kHz}$ and net damping rate $-\gamma / \omega_{0} \approx 0.34 \%$, is the best match with the experimental AE. The poloidal mode structure is plotted with the Alfvén continuum in the lab frame in Fig. 6a, with dominant couplings of poloidal harmonics $m=(9,11),(10,12)$, and $(11,13)$. Its localization around $\sqrt{\psi_{\mathrm{N}}} \approx 0.7-0.9$ $\left(\psi_{\mathrm{N}} \approx 0.5-0.8\right)$ is consistent with improved AEAD coupling to the edge as opposed to core

+ The contributions from ICRH-accelerated fast ions and ${ }^{3} \mathrm{He}$ ions are not included in the present work. 


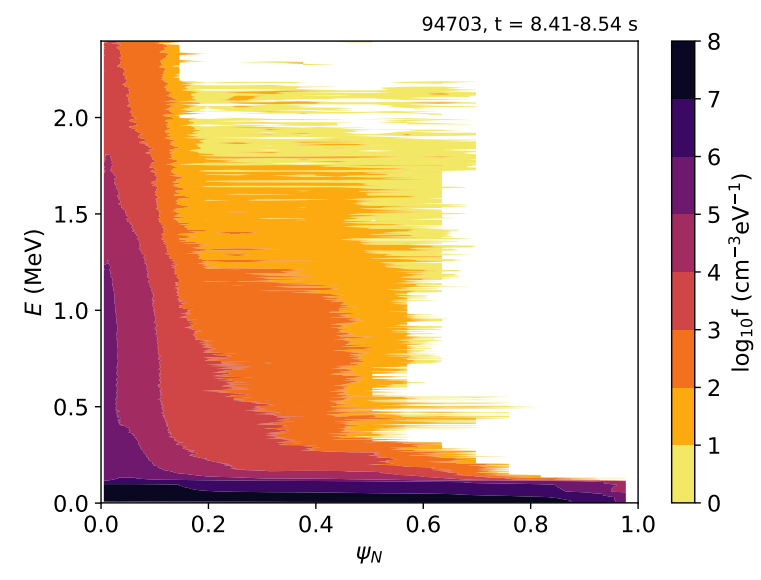

(a)

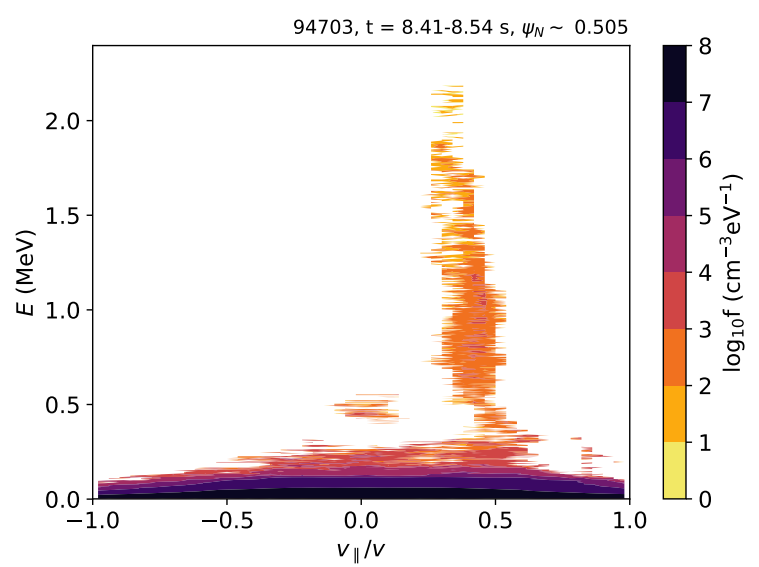

(b)

Figure 5: Deuterium fast ion distribution functions (logarithmic) from TRANSP for JPN 94703 and integrated over $t=8.41-8.54 \mathrm{~s}$ : (a) averaged over all pitches, and (b) at $\psi_{\mathrm{N}} \approx 0.5$.

Table 2: Normalized damping rate (\%) calculated from NOVA-K. The frequency is in the lab frame, and uncertainties of continuum damping are $\pm 0.1 \%$.

\begin{tabular}{lccc}
\hline Damping $\gamma / \omega_{0}(\%)$ & $n=3, f_{0}=243.1 \mathrm{kHz}$ & $n=5, f_{0}=236.4 \mathrm{kHz}$ & $n=6, f_{0}=232.7 \mathrm{kHz}$ \\
\hline Continuum & -0.092 & -0.116 & -0.177 \\
Radiative & 0.000 & 0.000 & 0.000 \\
Electron collisional & -0.011 & -0.010 & -0.010 \\
Electron Landau & -0.161 & -0.198 & -0.176 \\
Ion Landau & $\sim 0.000$ & $\sim 0.000$ & $\sim 0.000$ \\
NBI fast ions & -0.031 & -0.017 & -0.014 \\
Total & $\mathbf{- 0 . 2 9 5}$ & $\mathbf{- 0 . 3 4 1}$ & $\mathbf{- 0 . 3 7 8}$ \\
\hline
\end{tabular}

modes, which has also been observed in previous simulation work $[7,39,43]$.

The simulated $n=3$ mode is shown in Fig. $6 \mathrm{~b}$ for comparison. Dominant poloidal harmonics are $m=(5,7)$ and $(6,8)$. Note that the localization is similar to that of $n=5$. Thus, there is certainly some ambiguity in toroidal mode number of the measured AE. It could even be a superposition of modes. Nevertheless, NOVA-K is consistent in matching the frequency, net damping rate, and expected mode location.

From Table 2, the dominant contributions to the AE's marginal stability are electron Landau and continuum damping, with small contributions from NBI fast ion and electron collisional damping. The explanation for the dominance of electron over ion Landau damping is likely due to the relatively high Alfvén speed, $v_{\mathrm{A}} \approx 7 \times 10^{6} \mathrm{~m} / \mathrm{s}$, compared to the electron and $\mathrm{D}$ ion thermal speeds, $v_{\mathrm{te}} \approx 5.7 v_{\mathrm{A}}$ and $v_{\mathrm{ti}} \approx 0.1 v_{\mathrm{A}}$, respectively. The intersections of the candidate modes and the Alfvén continua are clearly seen in Fig. 6. The reason that the AEAD's magnetic perturbation was able to "tunnel through" the edge continuum to excite 


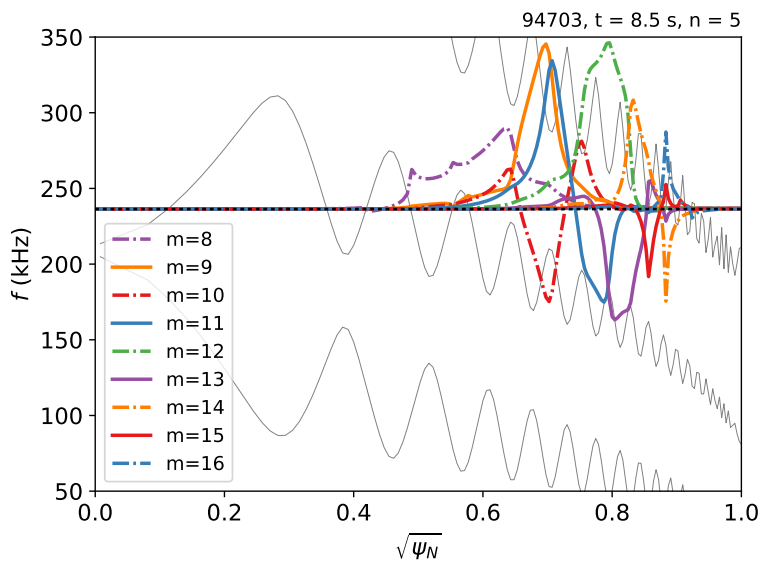

(a)

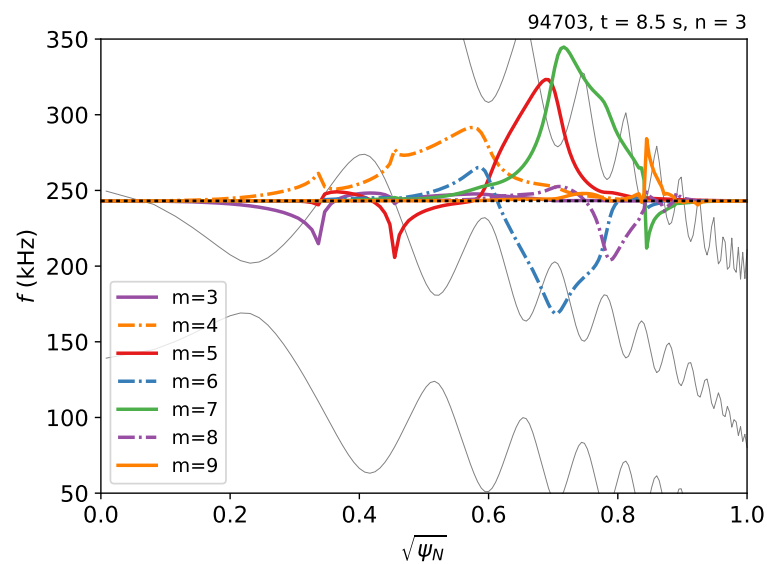

(b)

Figure 6: Continua (thin lines) and poloidal mode structure (solid, dot-dashed) from NOVA-K at $t=8.5 \mathrm{~s}$ for (a) $n=5$ and (b) $n=3$. The frequency (lab-frame) is indicated by the horizontal dotted line. $\psi_{\mathrm{N}}$ is the normalized poloidal flux.

this stable AE may simply be due to the small magnitude of the continuum damping, only $\left|\gamma / \omega_{0}\right| \approx 0.1 \%-0.2 \%$. This could help explain why only small damping rates are observed at high $n_{\mathrm{e} 95}$ in Fig. 2a.

The almost negligible damping from NBI fast ions makes sense since their parallel injection velocities, $v_{\|} \approx 1.6 \times 10^{6} \mathrm{~m} / \mathrm{s}$, are less than $v_{\mathrm{A}} / 2$, so there is little interaction with the candidate EAEs. Finally, note that the calculated radiative damping is totally negligible here. Thus, the damping mechanisms of this edge-localized EAE are very different from those of some core-localized TAEs in JET studied earlier with the AEAD [37] where radiative and ion Landau damping were dominant. Yet this is expected given the different AEs, localizations, and plasma scenarios.

Finally, we note that the Alfvén continua for this pulse (see Fig. 6) exhibit an interesting feature: the TAE and EAE gaps exist at almost the same frequency but over different radial ranges - core and edge, respectively - due, in part, to the differential plasma rotation (see Fig. 3b) and associated Doppler shift. Though not shown here, this result is confirmed with the Alfvén continuum solver CSCAS [56] using the magnetic geometry output from HELENA [57]. Such radially aligned gaps have been studied before - analytically and numerically - for reversed-shear $q$-profiles [58]; however, in that study, core EAE and edge TAE gaps were aligned, opposite to the present work.

In our case, it is reasonable to ask whether the AEAD is resonating with a core TAE instead of an edge EAE. ${ }^{*}$ A scan in the $q$-profile - i.e. a translation up/down by \pm 0.1 and

* Pursuing such TAE solutions is not without some experimental motivation: there are plasma discharges very similar to JPN 94703 and also part of JET's three-ion D-(D $\left.\mathrm{D}_{\mathrm{NBI}}\right)-{ }^{3} \mathrm{He}$ heating scenario experiments (JPN 95683, for example [47]), where core-localized TAEs were driven by energetic ions. Further analyses of these plasmas should be pursued, but are beyond the scope of the present work. 
\pm 0.2 - in NOVA-K finds no $n=3$ or 5 eigenmode solutions in the TAE gap close to the experimentally measured frequency. Importantly, the $n=5$ EAE is the most robust solution to this sensitivity study. However, by significantly lowering the central shear, an $n=3$ TAE solution appears near $\sqrt{\psi_{\mathrm{N}}} \approx 0.4$ with frequency $f_{0} \approx 235 \mathrm{kHz}$. This is consistent with earlier observations that the three-ion ICRH scenario can result in the flattening and even the reversal of the $q$-profile in the central regions of the plasma [47]. However, since such profiles are outside of experimental uncertainties here, we conclude that our measurement corresponds to the edge-localized EAE predicted by the most reasonable assumptions.

\section{Summary}

Understanding the interaction of Alfvén Eigenmodes (AEs) and energetic particles (EPs) is important to the success of future tokamaks. In JET, eight toroidally spaced, in-vessel antennas - collectively called the Alfvén Eigenmode Active Diagnostic (AEAD) - actively probe stable AEs with frequencies ranging $f=25-250 \mathrm{kHz}$ and toroidal mode numbers $|n|<20$. The AEAD plays an especially important role when AEs are not destabilized by EPs, which could even be the case for alpha drive in the upcoming JET DT campaign.

During the 2019-2020 deuterium campaign, $\sim 7500$ resonances - along with their frequencies $\omega_{0}=2 \pi f_{0}$, net damping rates $\gamma<0$, and toroidal mode numbers $n$ - were measured in $\sim 800$ plasma discharges. A statistical analysis was performed on the database: continuum and radiative damping were found to increase with edge safety factor, edge magnetic shear, and when including non-ideal effects (see Table 1 and Fig. 1a), as expected from theory. A lower bound on ion Landau damping from NBI fast ions was also inferred (see Fig. 1b).

By comparing the stable AE database with the EUROfusion JET-ILW pedestal database, the AEAD was determined to be inefficient in probing stable AEs during H-mode periods. One likely cause is reduced AEAD accessibility due to interactions with the edge Alfvén continuum. This is consistent with stable AE observations limited by high edge densities and steep edge density gradients (see Fig. 2a). Reduced signal-to-noise is also a factor as ELMs can inhibit the identification of stable AE resonances in the magnetics data. Finally, the number of stable AE measurements and their corresponding damping rates were found to decrease with $n$ (see Fig. $2 \mathrm{~b}$ ), possibly due to mode widths and damping becoming more localized.

A novel measurement was presented of a marginally stable EAE resonantly excited by the AEAD at the edge of an L-mode/L-mode plasma (JPN 94703) with high-power auxiliary heating, i.e. ICRH and NBI up to $25 \mathrm{MW}$ (see Figs. 3 and 5). This stable AE was tracked in real time with frequency $f_{0} \approx 235-250 \mathrm{kHz}$, net damping rate $-\gamma / \omega_{0} \approx 0.17 \%-0.45 \%$, and estimated toroidal mode number $|n| \approx 3-6$ (see Fig. 4).

NOVA-K kinetic-MHD simulations showed good agreement with experimental measurements, indicating the dominance of electron Landau and continuum damping for a marginally stable edge-localized EAE (see Table 2 and Fig. 6). These dominant contributions, 
as well as negligible contributions from thermal ion and NBI fast ion Landau damping, match physical intuition due to the relatively high Alfvén speed compared to thermal electron/ion and NBI parallel injection velocities. A scan of the $q$ profile within experimental uncertainties indicated that the $n=5$ edge EAE solution in JPN 94703 is robust, while a core/mid-radius TAE solution is unlikely in this case.

With this demonstration of a successful AE stability measurement by the AEAD in a deuterium plasma with high-power auxiliary heating, we are optimistic for similar measurements in the ongoing tritium and upcoming DT campaigns.

\section{Acknowledgments}

The authors thank V. Aslanyan, P. Bonofiglo, N. Dreval, L. Frassinetti, N. Gorelenkov, N. Hawkes, Ph. Lauber, S. Menmuir, E. Rachlew, E. Solano, G. Szepesi, A. Teplukhina, and D. Testa for their contributions to this paper. The authors are also grateful to the reviewers who helped improve this paper. This work was supported by US DOE grants DE-SC0014264 and DE-AC02-09CH11466, as well as the Brazilian agency FAPESP Project 2011/50773-0. This work has been carried out within the framework of the EUROfusion Consortium and has received funding from the Euratom research and training program 2014-2018 and 20192020 under grant agreement No 633053. The views and opinions expressed herein do not necessarily reflect those of the European Commission.

\section{References}

[1] A. Fasoli, D. Borba, G. Bosia, D. J. Campbell, J. A. Dobbing, C. Gormezano, J. Jacquinot, P. Lavanchy, J. B. Lister, P. Marmillod, J. M. Moret, A. Santagiustina, and S. Sharapov. Direct measurement of the damping of toroidicity-induced Alfvén eigenmodes. Physical Review Letters, 75(4):645-648, 1995.

[2] T. Panis, D. Testa, A. Fasoli, A. Klein, H. Carfantan, P. Blanchard, and JET-EFDA Contributors. Optimization of the active MHD spectroscopy system on JET for the excitation of individual intermediate and high-n Alfvén eigenmodes. Nuclear Fusion, 50(084019), 2010.

[3] P. Puglia, W. Pires de Sa, P. Blanchard, S. Dorling, S. Dowson, A. Fasoli, J. Figueiredo, R. Galvão, M. Graham, G. Jones, C. Perez von Thun, M. Porkolab, L. Ruchko, D. Testa, P. Woskov, M.A. Albarracin-Manrique, and JET Contributors. The upgraded JET toroidal Alfvén eigenmode diagnostic system. Nuclear Fusion, 56(11):112020, nov 2016.

[4] R. J. Dumont, J. Mailloux, V. Aslanyan, M. Baruzzo, C.D. Challis, I. Coffey, A. Czarnecka, E. Delabie, J. Eriksson, J. Faustin, J. Ferreira, M. Fitzgerald, J. Garcia, L. Giacomelli, C. Giroud, N. Hawkes, Ph. Jacquet, E. Joffrin, T. Johnson, D. Keeling, D. King, V. Kiptily, B. Lomanowski, E. Lerche, M. Mantsinen, L. Meneses, S. Menmuir, K. McClements, S. Moradi, F. Nabais, M. Nocente, A. Patel, H. Patten, P. Puglia, R. Scannell, S. Sharapov, E. R. Solano, M. Tsalas, P. Vallejos, H. Weisen, and JET Contributors. Scenario development for the observation of alpha-driven instabilities in JET DT plasmas. Nuclear Fusion, 58(8):082005, aug 2018.

[5] E. Joffrin et al. Overview of the JET preparation for deuterium-tritium operation with the ITER like-wall. Nuclear Fusion, 59(11):112021, aug 2019.

[6] R A Tinguely, P G Puglia, N Fil, S Dowson, M Porkolab, A Fasoli, D Testa, and Jet Contributors. Results from the Alfvén Eigenmode Active Diagnostic during the 2019- 2020 JET deuterium campaign. Plasma Phys. Control. Fusion, 62:11, 2020. 
[7] R A Tinguely, P G Puglia, N Fil, S Dowson, M Porkolab, A Dvornova, A Fasoli, M Fitzgerald, V Guillemot, G T A Huysmans, M Maslov, S Sharapov, and D Testa. Experimental studies of plasmaantenna coupling with the JET Alfvén Eigenmode Active Diagnostic. Nuclear Fusion, 61:26003-26017, 2021.

[8] A. Fasoli, J.B. Lister, S.E. Sharapov, S. Ali-Arshad, G. Bosia, D. Borba, D.J. Campbell, N. Deliyanakis, J.A. Dobbing, C. Gormezano, H.A. Holties, G.T.A. Huysmans, J. Jacquinot, A. Jaun, W. Kerner, P. Lavanchy, J.-M. Moret, L. Porte, A. Santagiustina, and L. Villard. Overview of Alfven eigenmode experiments in JET. Nucl. Fusion, 35:1485, 1995.

[9] A Fasoli, J B Lister, S Sharapov, D Borba, N Deliyanakis, C Gormezano, J Jacquinot, A Jaun, H A Holties, G T A Huysmans, W Kerner, J.-M Moret, and L Villard. Observation of Multiple Kinetic Alfvén Eigenmodes. Physical Review Letters, 76(7), 1996.

[10] A Fasoli, D Borba, C Gormezano, R Heeter, A Jaun, J Jacquinot, W Kerner, Q King, J B Lister, S Sharapov, D Start, and L Villard. Alfvén eigenmode experiments in tokamaks and stellarators. Plasma Phys. Control. Fusion, 39(1067):287-301, 1997.

[11] W W Heidbrink, A Fasoli, D Borba, and A Jaun. Stable ellipticity-induced Alfvén eigenmodes in the Joint European Torus. Physics of Plasmas, 4:3663, 1997.

[12] A. Jaun, A. Fasoli, and W. W. Heidbrink. Prediction of Alfvén eigenmode dampings in the Joint European Torus. Physics of Plasmas, 5(8):2952-2955, 1998.

[13] King-Lap Wong. A review of Alfvén eigenmode observations in toroidal plasmas. Plasma Physics and Controlled Fusion, 41(1):R1-R56, jan 1999.

[14] A. Fasoli, D. Borba, B. Breizman, C. Gormezano, R. F. Heeter, A. Juan, M. Mantsinen, S. Sharapov, and D. Testa. Fast particles-wave interaction in the Alfvén frequency range on the Joint European Torus tokamak. Physics of Plasmas, 7(5):1816-1824, may 2000.

[15] A. Fasoli, A. Jaun, and D. Testa. Isotope mass scaling of AE damping rates in the JET tokamak plasmas. Physics Letters, Section A: General, Atomic and Solid State Physics, 265(4):288-293, jan 2000.

[16] A Jaun, A Fasoli, and J Vaclavik. Gyrokinetic modelling of macro-instabilities in high performance tokamak plasmas. Plasma Phys. Control. Fusion, 43:207, 2001.

[17] D. Testa and A. Fasoli. The effect of plasma shaping on the damping of low-n Alfvén eigenmodes in JET tokamak plasmas. Nuclear Fusion, 41(7):809-812, jul 2001.

[18] A Fasoli, D Testa, S Sharapov, H L Berk, B Breizman, A Gondhalekar, R F Heeter, M Mantsinen, and contributors to the EFDA-JET Workprogramme. MHD spectroscopy. Plasma Physics and Controlled Fusion, 44(12B):B159-B172, dec 2002.

[19] D Testa, A Fasoli, A Jaun, Al, NN Gorelenkov, HL Berk, R Budny, C Boswell, and Marcos A Albarracin. Experimental test of damping models for $\mathrm{n}=1$ toroidal Alfvén eigenmodes in JET. Nuclear Fusion, 43(479):479-482, 2003.

[20] D Testa, A Fasoli, and A Jaun. Measurement of the damping rate of toroidal Alfvén eigenmodes as a function of the neutral beam heating power and plasma $\beta$ on JET. Nuclear Fusion, 43:724-728, 2003.

[21] D. Testa, A. Fasoli, and E. Solano. Diagnosis and study of Alfvén eigenmodes stability in JET (invited). Review of Scientific Instruments, 74(3 II):1694-1700, mar 2003.

[22] D Testa, C Boswell, A Fasoli, Al, D Borba, M de Baar, M Bigi, J Brzozowski, P de Vries, and JET-EFDA Contributors. Alfvén mode stability and wave-particle interaction in the JET tokamak: prospects for scenario development and control schemes in burning plasma experiments. Plasma Phys. Control. Fusion, 46:59-79, 2004.

[23] D. Testa, C. Boswell, and A. Fasoli. Experimental study of the dependence of the damping rate of $\mathrm{n} \leq$ 1 TAEs on the on-axis safety factor and toroidal rotation shear. Nuclear Fusion, 45(8):907-917, aug 2005.

[24] D Testa, C Boswell, A Fasoli, and JET-EFDA Contributors. Measurement of the instability threshold for toroidal Alfvén eigenmodes in JET plasmas with forward and reversed magnetic field. Nucl. Fusion, 46:829-836, 2006. 
[25] A. Fasoli, C. Gormenzano, H. L. Berk, B. Breizman, S. Briguglio, D. S. Darrow, N. Gorelenkov, W. W. Heidbrink, A. Jaun, S. V. Konovalov, R. Nazikian, J. M. Noterdaeme, S. Sharapov, K. Shinohara, D. Testa, K. Tobita, Y. Todo, G. Vlad, and F. Zonca. Chapter 5: Physics of energetic ions. Nuclear Fusion, 47(S264-S284):S264-S284, 2007.

[26] A. Klein, H. Carfantan, D. Testa, A. Fasoli, and J. Snipes. A sparsity-based method for the analysis of magnetic fluctuations in unevenly-spaced Mirnov coils. Plasma Physics and Controlled Fusion, 50(12):125005, 2008.

[27] A. Fasoli, D. Testa, T. Panis, A. Klein, J. A. Snipes, J. Sears, M. Gryaznevich, R. Martin, and S. D. Pinches. Active excitation and damping rate measurement of intermediate-n toroidal Alfvén eigenmodes in JET, C-Mod and MAST plasmas. Plasma Physics and Controlled Fusion, 52(7):075015, 2010.

[28] D Testa, N Mellet, T Panis, P Blanchard, H Carfantan, A Fasoli, and Jet-efda Contributors. The dependence of the damping rate of medium-n toroidal Alfven eigenmodes on the edge plasma elongation in JET. Nucl. Fusion, 50(16):084010, 2010.

[29] D. Testa, H. Carfantan, A. Goodyear, P. Blanchard, A. Klein, T. Panis, and JET-EFDA contributors. The JET Alfvén Eigenmode Local Manager for the real-time detection and tracking of MHD instabilities. A Letters Journal Exploring the Frontiers of Physics, 92(50001), 2010.

[30] D Testa, D Spong, T Panis, P Blanchard, A Fasoli, and Jet-efda Contributors. Recent JET experiments on Alfvén eigenmodes with intermediate toroidal mode numbers: measurements and modelling of $\mathrm{n}$ $=3$ toroidal Alfvén eigenmodes with the TAEFL code. Nucl. Fusion, 51:43009-43020, 2011.

[31] D. Testa, H. Carfantan, A. Fasoli, A. Goodyear, Q. King, P. Blanchard, A. Klein, P. Lavanchy, and T. Panis. The JET Alfvén Eigenmode Local Manager for the real-time detection and tracking of a frequency-degenerate spectrum of MHD instabilities. Fusion Engineering and Design, 86(4-5):381392, jun 2011.

[32] T Panis, A Fasoli, D Testa, and JET-EFDA Contributors. Analysis of damping rate measurements of toroidal Alfvén eigenmodes on JET as a function of n: part I. Nucl. Fusion, 52(3):023013, 2012.

[33] T. Panis, A. Fasoli, and D. Testa. Analysis of damping rate measurements of toroidal Alfvén eigenmodes as a function of n: Part II. Nuclear Fusion, 52(3):023014, 2012.

[34] D Testa, T Panis, P Blanchard, A Fasoli, and Jet-efda Contributors. Plasma isotopic effect on the damping rate of toroidal Alfvén eigenmodes with intermediate toroidal mode numbers. Nuclear Fusion, 52:094006, 2012.

[35] Duccio Testa, Hervé Carfantan, and Alex Goodyear. Implementation of a novel real-time controller for the detection and tracking of magneto-hydrodynamic instabilities on the JET tokamak. Plasma and Fusion Research, 9(1405003):1-46, 2014.

[36] F. Nabais, V. Aslanyan, D. Borba, R. Coelho, R. Dumont, J. Ferreira, A. Figueiredo, M. Fitzgerald, E. Lerche, J. Mailloux, M. Mantsinen, P. Rodrigues, M. Porkolab, P. Puglia, S.E. Sharapov, and JET Contributors. TAE stability calculations compared to TAE antenna results in JET. Nuclear Fusion, 58(8):082007, aug 2018.

[37] V. Aslanyan, S. Taimourzadeh, L. Shi, Z. Lin, G. Dong, P. Puglia, M. Porkolab, R. Dumont, S. E. Sharapov, J. Mailloux, M. Tsalas, M. Maslov, A. Whitehead, R. Scannell, S. Gerasimov, S. Dorling, S. Dowson, H. K. Sheikh, T. Blackman, G. Jones, A. Goodyear, K. K. Kirov, P. Blanchard, A. Fasoli, and D. Testa. Gyrokinetic simulations of toroidal Alfvén eigenmodes excited by energetic ions and external antennas on the Joint European Torus. Nuclear Fusion, 59(2), feb 2019.

[38] M Saigusa, Y Kusama, T Ozeki, H Kimura, T Fujita, S Moriyama, T Fujii, M Azumi, V.I Afanassiev, Y Neyatani, G.Y Fu, and C.Z Cheng. Effect of shear in toroidal rotation on toroidicity induced Alfvén eigenmodes. Nuclear Fusion, 37(11):1559-1568, nov 1997.

[39] A. Dvornova, G. T. A. Huijsmans, S. Sharapov, F. J. Artola Such, P. Puglia, M. Hoelzl, S. Pamela, A. Fasoli, and D. Testa. Modeling of TAE mode excitation with an antenna in realistic X-point geometry. Physics of Plasmas, 27(1):012507, jan 2020.

[40] W. W. Heidbrink. Basic physics of Alfvén instabilities driven by energetic particles in toroidally confined 
plasmas. Physics of Plasmas, 15(5):055501, may 2008.

[41] R. R. Mett and S. M. Mahajan. Kinetic theory of toroidicity-induced Alfvén eigenmodes. Physics of Fluids B, 4(9):2885-2893, sep 1992.

[42] R R Mett, E J Strait, and S M Mahajan. Damping of toroidal Alfvén modes in DIII-D. Physics of Plasmas, 1:3277, 1994.

[43] D. Borba, A. Fasoli, N.N. Gorelenkov, S. Gunter, Ph. Lauber, N. Mellet, R. Nazikian, T. Panis, S.D. Pinches, D. Spong, D. Testa, and JET-EFDA Contributors. The Influence of Plasma Shaping on the Damping of Toroidal Alfvén Eigenmodes. 23rd IAEA Fusion Energy Conference, (THW/P7-08):1-8, 2010.

[44] L Frassinetti, S Saarelma, G Verdoolaege, M Groth, J C Hillesheim, P Bilkova, P Bohm, M Dunne, R Fridström, E Giovannozzi, F Imbeaux, B Labit, E De La Luna, C Maggi, M Owsiak, and R Scannell. Pedestal structure, stability and scalings in JET-ILW: the EUROfusion JET-ILW pedestal database. Nuclear Fusion, 61(016001), 2021.

[45] Ye O Kazakov, Massimo Nocente, MJ Mantsinen, Jozef Ongena, Yuriy Baranov, Teddy Craciunescu, Mykola Dreval, Remi Dumont, Jacob Eriksson, Jeronimo Garcia, et al. Plasma heating and generation of energetic D ions with the 3-ion ICRF+ NBI scenario in mixed HD plasmas at JET-ILW. Nuclear Fusion, 60(11):112013, 2020.

[46] Massimo Nocente, Ye O Kazakov, J Garcia, VG Kiptily, Jozef Ongena, Mykola Dreval, Michael Fitzgerald, Sergei E Sharapov, Z Stancar, Henri Weisen, et al. Generation and observation of fast deuterium ions and fusion-born alpha particles in JET plasmas with the 3-ion radio-frequency heating scenario. Nuclear Fusion, 60(12):124006, 2020.

[47] Ye. O. Kazakov, J. Ongena, J. C. Wright, S. J. Wukitch, V. Bobkov, J. Garcia, V. G. Kiptily, M. J. Mantsinen, M. Nocente, M. Schneider, H. Weisen, Y. Baranov, M. Baruzzo, R. Bilato, A. Chomiczewska, R. Coelho, T. Craciunescu, K. Crombé, M. Dreval, R. Dumont, P. Dumortier, F. Durodié, J. Eriksson, M. Fitzgerald, J. Galdon-Quiroga, D. Gallart, M. Garcia-Muñoz, L. Giacomelli, C. Giroud, J. Gonzalez-Martin, A. Hakola, P. Jacquet, T. Johnson, A. Kappatou, D. Keeling, D. King, K. K. Kirov, P. Lamalle, M. Lennholm, E. Lerche, M. Maslov, S. Mazzi, S. Menmuir, I. Monakhov, F. Nabais, M. F. F. Nave, R. Ochoukov, A. R. Polevoi, S. D. Pinches, U. Plank, D. Rigamonti, M. Salewski, P. A. Schneider, S. E. Sharapov, Ž. Štancar, A. Thorman, D. Valcarcel, D. Van Eester, M. Van Schoor, J. Varje, M. Weiland, and N. Wendler. Physics and applications of three-ion icrf scenarios for fusion research. Physics of Plasmas, 28(2):020501, 2021.

[48] L.L. Lao, H. St. John, R.D. Stambaugh, A.G. Kellman, and W. Pfeiffer. Reconstruction of current profile parameters and plasma shapes in tokamaks. Nuclear Fusion, 25(11):1611-1622, 1985.

[49] Joshua Breslau, Marina Gorelenkova, Francesca Poli, Jai Sachdev, and Xingqiu Yuan. TRANSP. [Computer Software] https://doi.org/10.11578/dc.20180627.4, jun 2018.

[50] RJ Hawryluk. An empirical approach to tokamak transport. In Coppi et al, editor, Physics Close to Thermonuclear Conditions, Ed. B, volume 19. Brussels: Commission of the European Communities, Brussels, 1980.

[51] JPHE Ongena, I Voitsekhovitch, M Evrard, and D McCune. Numerical transport codes. Fusion Science and Technology, 61(2T):180-189, 2012.

[52] Emilia R. Solano, N. Vianello, E. Delabie, J.C. Hillesheim, P. Buratti, D. Réfy, I. Balboa, A. Boboc, R. Coelho, B. Sieglin, S. Silburn, P. Drewelow, S. Devaux, D. Dodt, A. Figueiredo, L. Frassinetti, S. Marsen, L. Meneses, C.F. Maggi, J. Morris, S. Gerasimov, M. Baruzzo, M. Stamp, D. Grist, I. Nunes, F. Rimini, S. Schmuck, I. Lupelli, and C. Silva and. Axisymmetric oscillations at L-H transitions in JET: M-mode. Nuclear Fusion, 57(2):022021, oct 2017.

[53] CZ Cheng. Kinetic extensions of magnetohydrodynamics for axisymmetric toroidal plasmas. Physics Reports, 211(1):1-51, 1992.

[54] GY Fu and CZ Cheng. Excitation of high-n toroidicity-induced shear Alfvén eigenmodes by energetic particles and fusion alpha particles in tokamaks. Physics of Fluids B: Plasma Physics, 4(11):3722$3734,1992$. 
[55] NN Gorelenkov, Chio-Zong Cheng, and GY Fu. Fast particle finite orbit width and Larmor radius effects on low-n toroidicity induced Alfvén eigenmode excitation. Physics of Plasmas, 6(7):2802-2807, 1999.

[56] G. T. A. Huysmans, S. E. Sharapov, A. B. Mikhailovskii, and W. Kerner. Modeling of diamagnetic stabilization of ideal magnetohydrodynamic instabilities associated with the transport barrier. Physics of Plasmas, 8(10):4292-4305, 2001.

[57] G.T.A. Huysmans, J.P. Goedbloed, and W. Kerner. Isoparametric Bicubic Hermite Elements for Solution of the Grad-Shafranov Equation. International Journal of Modern Physics C, 02(01):371376, 1991.

[58] N. N. Gorelenkov. Double-gap alfvén eigenmodes: Revisiting eigenmode interaction with the alfvén continuum. Phys. Rev. Lett., 95:265003, Dec 2005. 